\title{
Space Hierarchical Design of Mobile User Interface Based on Eye Movement Experiment
}

\author{
Jing $\operatorname{Jin}^{1, a^{*}}$, Huaqing Shen ${ }^{2, b}$ \\ ${ }^{1}$ School of Humanities, Zhejiang University, Hangzhou, Zhejiang 310012, China \\ ${ }^{2}$ School of Humanities, Zhejiang University, Hangzhou, Zhejiang 310012, China \\ a313622091@qq.com, bsky_0120123@163.com
}

\begin{abstract}
Keywords: Space hierarchy, Eye movement experiment
Abstract. In this paper, the idea of UI space hierarchy of mobile terminal was put forward and the UI space was divided into four levels. Taking iPhone5 as an example, RGB, angle, opacity, size and other regional shadow parameter values were set first of all and then representative sample combination was selected by orthogonal experimental design, subjects need to perceptual image evaluate the sample pictures through the eye movement experiment and the eye movement experimental data was analyzed by the method of orthogonal analysis. The results proved the existence of UI space hierarchy, and the best UI space shadow hierarchy combination regimen was summed up. The results have theoretical and practical value to the study of UI interface design.
\end{abstract}

\section{Introduction}

With the development of network technology, the mobile terminal interface design needs to reflect the elements of fashion trend, meet the characteristics of human culture and have affinity. The mobile terminal interface should not only have high picture quality, but also need more personalized recognition and operational process[1].

Interface design is a complex project which has different subjects to participate in and the criterion for testing an interface is the feeling and experience of the end user[2]. Similar studies at home and abroad were often limited to subjective feeling before, which were lack of objective evaluation, rarely referring to the study of UI space hierarchy of mobile terminal. Based on the eye movement experiment method of cognitive psychology, this paper studied the UI space hierarchy of mobile terminal and selected representative sample combination combined with orthogonal experiment method. The data was comprehensively analyzed from subjective and objective aspects, which makes up for the lack of objective data analysis in such studies and makes the conclusion more objective and reliable. The research can provide reference for mobile terminal interaction design.

\section{The idea and element of UI space hierarchy of mobile terminal}

\section{The idea of UI space hierarchy of mobile terminal}

Since the electronic screens of mobile terminal are completely complanation, which is not $3 \mathrm{~d}$ as in the reality. This study aims to put the characteristics of real paper into electronic screens and present the information content in the virtual paper which has the upper and lower hierarchy relationship with another paper (information content). The projection was used to simulate the spatial traits of the paper[3], which is shown in Fig. 1.

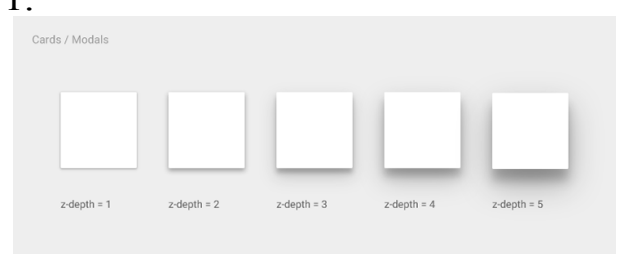

Fig. 1 Shade expression of cards 


\section{The elements of UI space hierarchy of mobile terminal}

As shown in Fig. 2, this study chose iPhone5 with IOS mobile operating system to research the user interface.

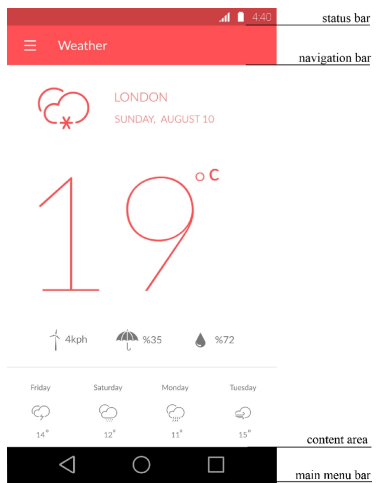

(1) The size and resolution of iphone5

Fig. 2 Example of APP interface

iPhone5 interface dimension: $640 \times 1136,72 \mathrm{ppi}$ is usually used in both website UI and mobile terminal UI.

(2) Basic element of interface

$640 \times 1136$ was chosen for the size design of which the element sizes are as follows: The size of status bar was 40 pixels, the size of navigation bar was 88 pixels, the size of main menu bar was 98 pixels and the size of content area was 910 pixels. The color was white, so the RBG values were R: 255, G: 255, B: 255.

(3) Shadow hierarchy design

We can get the shadow value of each area by setting the parameters (RGB values, angles, opacity, and size) in Photoshop software. In order to avoid the influence of color on the shadow hierarchical design, color was uniformly set as black, so the RGB values were R: 0, G: 0, B: 0. Angle was divided into $-90^{\circ}$ and $90^{\circ}$. The numerical range of opacity was from $20 \%$ to $70 \%$. The numerical range of size was from 10 pixels to 60 pixels.

\section{UI space hierarchical design experiment of mobile terminal}

This study selected representative samples from many compositions by the orthogonal experiment. Photoshop was used to make the sample image pictures, subjects need to perceptual image evaluate the sample pictures through the eye movement experiment and the eye movement experimental data was analyzed by mathematical analysis to discover the influence factors and the best scheme for UI space hierarchical design of mobile terminal.

\section{Orthogonal experimental design of UI space hierarchy}

Orthogonal experimental design is a design method to study multiple factor and level. It selects partial typical sample combinations from comprehensive experiment according the orthogonality, of which the combinations are usually representative and scientific[4].

Analyzing the designing elements of UI space hierarchy of mobile terminal, factors and levels table for orthogonal array design is established, in which there are nine factors, three rows with four levels and six rows with two levels. As shown in table 1, in order to simplify the name of factors, the shadow position between status bar and navigation bar was called position 1, the shadow position between navigation bar and main menu bar was called position 2, and the shadow position between main menu bar and content area was called position 3 .

Table 1 Factors and levels for L16 $\left(4^{3} \times 2^{6}\right)$ orthogonal array design

\begin{tabular}{|c|c|c|c|c|c|c|c|c|c|c|}
\hline level & factor & $\begin{array}{c}\text { Position1 } \\
\text { opacity } \\
(\%)\end{array}$ & $\begin{array}{l}\text { Position2 } \\
\text { opacity } \\
(\%)\end{array}$ & $\begin{array}{c}\text { Position3 } \\
\text { opacity } \\
(\%)\end{array}$ & $\begin{array}{l}\text { Position1 } \\
\text { size } \\
\text { (pixels) }\end{array}$ & $\begin{array}{l}\text { Position2 } \\
\text { size } \\
\quad \text { (pixels) }\end{array}$ & $\begin{array}{l}\text { Position3 } \\
\text { size } \\
\quad \text { (pixels) }\end{array}$ & $\begin{array}{l}\text { Position1 } \\
\text { angle } \\
\left({ }^{\circ}\right)\end{array}$ & $\begin{array}{l}\text { Position2 } \\
\text { angle } \\
\left({ }^{\circ}\right)\end{array}$ & $\begin{array}{l}\text { Position3 } \\
\text { angle } \\
\left({ }^{\circ}\right)\end{array}$ \\
\hline & mark & $\mathrm{A}$ & $\mathrm{B}$ & $\mathrm{C}$ & $\mathrm{D}$ & $\mathrm{E}$ & $\mathrm{F}$ & $\mathrm{G}$ & $\mathrm{H}$ & $\mathrm{I}$ \\
\hline 1 & & A1(20) & B1(40) & $\mathrm{C} 1(60)$ & D1(10) & E1(30) & F1(50) & G1(-90) & $\mathrm{H} 1(-90)$ & I1(-90) \\
\hline 2 & & A2(25) & B2(45) & $\mathrm{C} 2(65)$ & D2(20) & E2(40) & F2(60) & G2(90) & $\mathrm{H} 2(90)$ & $\mathrm{I} 2(90)$ \\
\hline 3 & & A3(30) & B3(50) & C3(70) & & & & & & \\
\hline 4 & & A4(35) & B4(55) & C4(75) & & & & & & \\
\hline
\end{tabular}




\section{Eye movement experiment}

\section{Equipment and programming}

(1) Equipment

Canada SR company of Eyelink1000 desktop eye tracking system production was used in this experiment. Eye tracking technology was used to test interface hierarchy layout and evaluate the designing schemes of UI space hierarchy.

(2) Programming

The EB programming of EyeLink1000 is a kind of modular and visual programming. While programming we get the basic frame by dragging the controls to the panel and connect them according to their experiment logical sequence by arrows[5].

\section{Subjects}

There are 55 subjects in the experiment, of which are 25 males and 30 females. The subjects are the college students or graduates majored in interaction or visual design. The eyesight of all subjects (naked or in glasses) was required to reach 1.0 and above vision.

\section{Method}

16 kinds of UI space hierarchy design combining scheme got from orthogonal experiment were made into sample image pictures by Photoshop. Guides for the typical tasks which subjects need to complete in the experiment are as follows: The first step is to observe which area of the picture is most obvious according to the first feeling and observe in turn; The second step is to observe the transition of three positions of shadow; The third step, if the subjects think they can't find fixation point or the fixation point is not obvious in the first two steps, they can press the space key to pass.

\section{Data extraction}

On rare cases in the process of the experiment, the related data of eye movement indicators will appear to be lack of records. 50 valid subject samples were obtained after examining the data records of all subjects' eye movement indicators and deleting some invalid samples which have much default value and vision loss.

\section{Experimental data analysis}

\section{Eye movement experiment data analysis}

The experimental data consists of two parts: fixation duration and fixation count. By classifying and average processing the output of excel report, the total fixation time data of 50 subjects for different hierarchical area of 16 pieces of image pictures was obtained.

\section{Orthogonal experiment data analysis}

The average of total fixation duration $(\mathrm{K})$ in the same level was calculated by orthogonal analysis and is shown in table 2 . 
Table 2 The results of orthogonal experiment data analysis(s)

\begin{tabular}{|c|c|c|c|c|c|c|c|c|c|c|}
\hline Factor & 1 & 2 & 3 & 4 & 5 & 6 & 7 & 8 & 9 & Results \\
\hline Number & $\mathrm{A}$ & $\mathrm{B}$ & $\mathrm{C}$ & $\mathrm{D}$ & $\mathrm{E}$ & $\mathrm{F}$ & G & $\mathrm{H}$ & I & Total fixation duration \\
\hline 1 & 1 & 1 & 1 & 1 & 1 & 1 & 1 & 1 & 1 & 42.85 \\
\hline 2 & 1 & 2 & 2 & 1 & 1 & 2 & 2 & 2 & 2 & 63.88 \\
\hline 3 & 1 & 3 & 3 & 2 & 2 & 1 & 1 & 2 & 2 & 65.96 \\
\hline 4 & 1 & 4 & 4 & 2 & 2 & 2 & 2 & 1 & 1 & 72.31 \\
\hline 5 & 2 & 1 & 2 & 2 & 2 & 1 & 2 & 1 & 1 & 56.42 \\
\hline 6 & 2 & 2 & 1 & 2 & 2 & 2 & 1 & 2 & 2 & 50.23 \\
\hline 7 & 2 & 3 & 4 & 1 & 1 & 1 & 2 & 2 & 2 & 66.62 \\
\hline 8 & 2 & 4 & 3 & 1 & 1 & 2 & 1 & 1 & 1 & 60.54 \\
\hline 9 & 3 & 1 & 3 & 1 & 2 & 1 & 2 & 1 & 2 & 59.08 \\
\hline 10 & 3 & 2 & 4 & 1 & 2 & 2 & 1 & 2 & 1 & 52.5 \\
\hline 11 & 3 & 3 & 1 & 2 & 1 & 1 & 2 & 2 & 1 & 43 \\
\hline 12 & 3 & 4 & 2 & 2 & 1 & 2 & 1 & 1 & 2 & 50.58 \\
\hline 13 & 4 & 1 & 4 & 2 & 1 & 1 & 1 & 1 & 2 & 70.46 \\
\hline 14 & 4 & 2 & 3 & 2 & 1 & 2 & 2 & 2 & 1 & 54.15 \\
\hline 15 & 4 & 3 & 2 & 1 & 2 & 1 & 1 & 2 & 1 & 67.46 \\
\hline 16 & 4 & 4 & 1 & 1 & 2 & 2 & 2 & 1 & 2 & 74.58 \\
\hline K1 & 61.25 & 57.2 & 52.67 & 60.94 & 56.51 & 58.98 & 57.57 & 60.85 & 56.15 & \\
\hline $\mathrm{K} 2$ & 58.45 & 55.19 & 59.59 & 57.89 & 62.32 & 59.85 & 61.26 & 57.98 & 62.67 & \\
\hline K3 & 51.29 & 60.76 & 59.93 & & & & & & & \\
\hline K4 & 66.66 & 64.5 & 65.47 & & & & & & & \\
\hline Range & 15.37 & 9.31 & 12.8 & 3.05 & 5.81 & 0.87 & 3.69 & 2.87 & 6.52 & \\
\hline Optimal case & A4 & B4 & $\mathrm{C} 4$ & D1 & E2 & $\mathrm{F} 2$ & G2 & H1 & $\mathrm{I} 2$ & \\
\hline
\end{tabular}

By calculating the average $(\mathrm{K})$ of total fixation time in the same level, we can get the maximum average of total fixation time of the same level, so this level of factor is the optimal level. For example, the maximum average of total fixation time of the factor $\mathrm{A}$ is $\mathrm{K} 4$, therefore $\mathrm{A} 4$ is the optimal level for the opacity of position 1.

To sum up, the best combination schemes of UI space shadow hierarchy is: Position 1 with the $35 \%$ opacity, 10 pixels size and $90^{\circ}$, Position 2 with the $55 \%$ opacity, 40 pixels size and $-90^{\circ}$ and Position 3 with the $75 \%$ opacity, 60 pixels size and $90^{\circ}$. The effect of the best combination schemes is shown in Fig. 3.

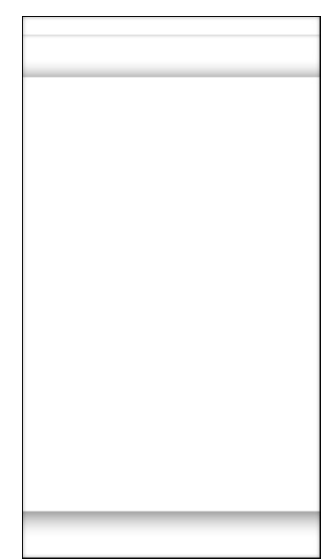

Fig. 3 The best combination scheme for UI space shadow hierarchy

\section{Conclusion}

The former interface design usually adjusts parameters only by designer's experience and feeling of which the interaction design can't meet the feeling and experience of the end user. However, The UI of mobile terminal is a direct dialogue between product and user. The design grasping the user will greatly improve the user experience of product. This research is a constant process to design satisfying visual effects for the end user, and produces interaction relationship with interface design by researching user experience.

This study researched the UI space hierarchy of mobile terminal by eye tracking experiment, orthogonal experiment and so on. And this paper got the best scheme for UI space hierarchy and discussed the factors influencing the UI space hierarchy. Several conclusions are presented as follows: 
(1) This study proved the idea of UI space hierarchy through the experiment and quantified and implemented the UI space hierarchy.

(2) This study summarized the best combination scheme of UI space shadow hierarchy, and its design parameters are as follows: Position 1 with the $35 \%$ opacity, 10 pixels size and $90^{\circ}$, Position 2 with the $55 \%$ opacity, 40 pixels size and $-90^{\circ}$ and Position 3 with the $75 \%$ opacity, 60 pixels size and $90^{\circ}$. which has a guideline value for UI design.

\section{References}

[1] Jianhua Meng, UI design and application, J. Artistic life, 4 (2009) 49-50.

[2] Meng Xia, Introduction to the UI design, J. Computer knowledge and technology, 7 (2015) 295-296.

[3] Information on http://www.uisdc.com/material-design-learning-experience.com

[4] Xiaobo Dai, Huiyuan Guan, The orthogonal experience design method in the application of the furniture product modeling design, J. Furniture, 3 (2013) 63-64.

[5] Jingjian $\mathrm{Xu}$, For Eyelink and Tobii eye instruments measuring performance comparison experiment, East China Normal University, 2009, pp. 3-4. 